\title{
Signo del halo reverso
}

\author{
Reverse halo sign
}

Jorge Alberto Carrillo, Paulina Ojeda, Juan Mauricio Pardo

- Bogotá, D.C.

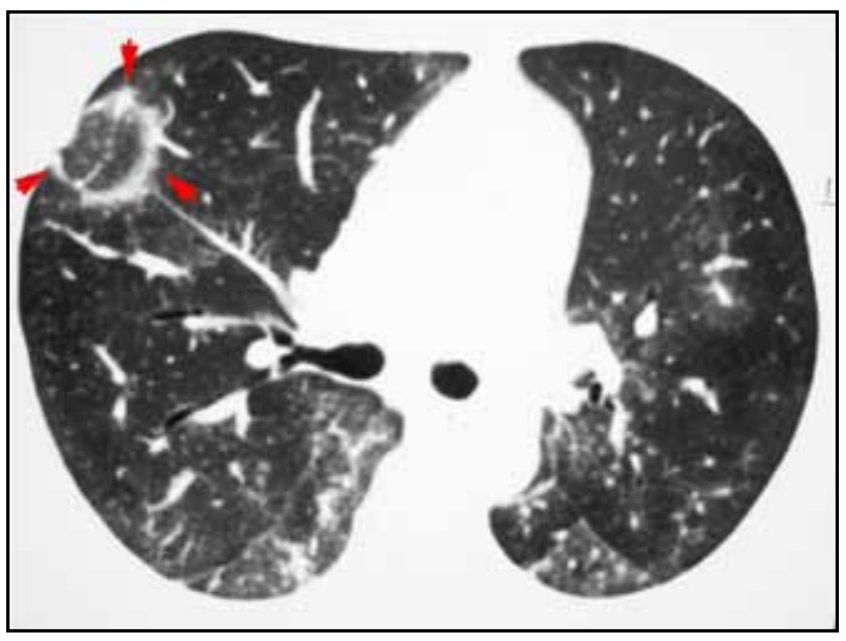

Paciente de 59 años con cuadro clínico de cuatro meses de evolución de pérdida de peso, fiebre, tos seca y disnea.

En la tomografía computarizada se evidenció una lesión nodular con densidad de "vidrio esmerilado" rodeada por un anillo de mayor densidad, asociada a áreas de aumento en la densidad del parénquima pulmonar con patrón en "vidrio esmerilado" y bronquios dilatados con pared engrosada. La biopsia pulmonar demostró pólipos de tejido fibroso en el espacio aéreo distal (flecha verde) e infiltrado intersticial crónico inflamatorio (flecha amarilla). Estos hallazgos son compatibles con el patrón histopatológico de neumonía de organización. Sin causa conocida, ni un contexto clínico específico asociado, se diagnosticó neumonía criptogénica de organización.

La sociedad Fleishner define el signo del halo reverso como un área focal redondeada con densidad de "vidrio esmerilado", rodeada por un anillo más o menos completo de consolidación. Este signo fue descrito inicialmente en pacientes con neumonía criptogénica de organización por Voloudaki (1) y $\operatorname{Kim}(2)$

Ha sido descrito en: 1) enfermedades infecciosas (paracoccidioidomicosis, aspergilosis, mucormicosis, 2) enfermedades linfoproliferativos (granulomatosis linfomatoidea), y 3 ) enfermedades inflamatorias no infecciosas ni neoplásicas (síndrome de Churg-Strauss, neumonía intersticial no específica y granulomatosis de Wegener).

\section{Referencias}

1. Voloudaki AE, Bouros DA, Froudarakis ME, Datseris GE,Apostolaki EG, Gourtsoyiannis NC. Crescentic and ring-shaped opacities. CT features in two cases of bronchiolitis obliterans organizing pneumonia (BOOP). Acta Radiol 1996;37:889-92.

2. Kim SJ, Lee KS, Ryu Y, Yoon Y, Choe K, Kim TS, Sung KJ. Reversed Halo Sign on High-Resolution CT of Cryptogenic Organizing Pneumonia: Diagnostic Implications. AJR Am J Roentgenol 2003;180:1251-4.
Dr. Jorge Alberto Carrillo B.: Médico Radiólogo, Méderi, Universidad del Rosario; Dra. Paulina Ojeda L: Patóloga, Hospita de Santa Clara; Dr. Juan Mauricio Pardo Oviedo: Internista, Jefe de Educación Médica, Méderi, Universidad del Rosario. Bogotá, D.C.

Correspondencia. Dr. Juan Mauricio Pardo E-mail: Juan.pardo@urosario.edu.co Recibido: 16/II/10 Aceptado: 16/II/10 